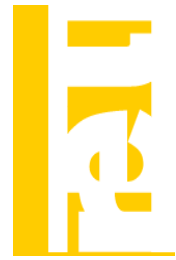

\title{
LAS DIFERENCIAS ENTRE EL PARO REGISTRADO Y EL PARO EPA EN GALICIA: UNA EVALUACIÓN CON MICRODATOS
}

\author{
Melchor Fernández ${ }^{1}$ y Manuel Flores
}

Universidad de Santiago de Compostela - IDEGA

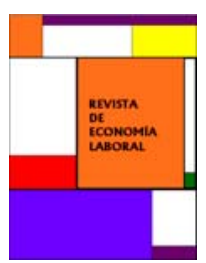

Recibido 22 Junio 2012; aceptado 8 Noviembre 2012

\section{Resumen}

Desde el inicio de la crisis, el paro estimado por la Encuesta de Población Activa (EPA) y el paro registrado (PR), pese a mostrar la misma tendencia, presentan divergencias muy significativas (por ejemplo, en el segundo trimestre de 2012 el paro EPA supera en más de un millón al PR para el conjunto de España). Este hecho, sin embargo, no se cumple en el caso de Galicia, donde son las cifras del PR las que superan ampliamente a las del paro EPA. El objetivo de este artículo es comprobar la validez de las cifras estimadas por la EPA para esta comunidad a partir de la explotación de los microdatos de una encuesta realizada a los demandantes de empleo en 2008.

Palabras clave: Desempleo, medición, análisis regional.

Clasificación JEL: J64, C81, R10.

\section{Abstract}

Since the beginning of the economic crisis, the unemployment series of the Labor Force Survey (LFS) and of the registered unemployment (RU) show both a similar trend of rapid employment destruction. But they show striking level differences as well (for instance, in the second quarter of 2012 the LFS unemployment exceeded by more than one million the $R U)$. This situation, however, does not apply to the region of Galicia, where the number of $R U$ usually exceeds the number offered by the LFS. The aim of the present study is to test the validity of the LFS estimates in this particular region by exploiting the microdata from a unique survey of job seekers in 2008.

Key words: Unemployment, measurement, regional analysis.

JEL Classification: J64, C81, R10.

\footnotetext{
1 Autor para correspondencia: melchor.fernandez@usc.es. Los autores agradecen los comentarios de los miembros del Grupo de Análisis y Modelización Económica de la Universidad de Santiago de Compostela a versiones preliminares del trabajo y, especialmente, los del editor y el evaluador de la revista, que conjuntamente han permitido mejorar sensiblemente el trabajo. Manuel Flores agradece la financiación recibida a través del programa predoctoral María Barbeito de la Xunta de Galicia. Lógicamente, los errores subsistentes son de completa responsabilidad de los autores.
}

(C) Revista de Economía Laboral 


\section{Introducción}

El diagnóstico de la situación económica de un país o región se realiza en base a una batería de indicadores entre los que destacan aquellos relacionados con el mercado laboral por su trascendencia política, económica y social. Conocer el número de parados y su evolución es un tema prioritario, ya que sin una estimación precisa es muy complicado establecer medidas para actuar sobre el desempleo. Para ello es imprescindible que nos pongamos de acuerdo sobre los requisitos que debe cumplir una persona parada. No hay métrica sin una rigurosa delimitación de lo que queremos medir, y en este punto debemos darnos cuenta de que no hay una población desempleada en abstracto.

En Galicia, como en el conjunto de España, hay dos estadísticas oficiales que miden periódicamente la evolución del paro: la Encuesta de Población Activa (EPA) que elabora el Instituto Nacional de Estadística (INE) y el paro registrado (PR) en las oficinas públicas de empleo que publica el Servicio Público de Empleo Estatal (SPEE). Estos instrumentos de medición dan resultados dispares en cuanto a la cifra total de desempleados, lo que se atribuye tanto a diferencias en la definición de desempleo como a diferencias metodológicas en su cálculo. En el segundo trimestre de 2012, la estimación del desempleo EPA supera en más de un millón de personas la cifra del PR. Al margen de interpretaciones metodológicas, lo cierto es que la existencia de dos cifras, en muchas ocasiones muy diferentes, resulta desconcertante si se hace la interpretación simplificada de que ambas miden con exactitud el mismo colectivo, es decir, el número de desempleados que realmente hay. En general, se considera que la cifra de desempleo registrado en las oficinas públicas de empleo será menor que el desempleo estimado a partir de la encuesta de fuerza de trabajo, ya que las condiciones para el registro suelen ser más restrictivas. No obstante, la Comisión Europea (1999) reconoce que el signo de esta relación depende completamente de las regulaciones nacionales, por lo que cualquier resultado es posible, como confirma el trabajo de Africa y Lüdeke (2006). Pero, aunque seamos capaces de justificar metodológicamente las discrepancias agregadas, las relaciones de signo opuesto entre ambas medidas del desempleo entre Comunidades Autónomas (CC.AA.) que comparten una misma legislación laboral exigen explicaciones adicionales.

Esta confusión de cifras ni es una novedad ni es exclusiva de España. En la mayoría de los países de la Unión Europea (UE) se publican oficialmente al menos dos cifras de desempleo, la que proviene de sus registros en las oficinas públicas de empleo y la obtenida a partir de una 
encuesta de fuerza de trabajo que cumple con las definiciones y criterios internacionales acordados por la Organización Internacional del Trabajo (OIT). Las discrepancias son importantes en casi todos los países, lo que ha generado un importante número de trabajos que tratan de buscar las razones de su existencia ${ }^{2}$.

Desde el punto de vista de la medición global del desequilibrio económico entre oferta y demanda en el mercado de trabajo, así como para el conocimiento de las características de la población activa y su evolución, resulta incontestable el superior grado de fiabilidad de las cifras que provienen de las encuestas de fuerza de trabajo como es el caso de la EPA. De hecho, el desempleo estimado a partir de esta encuesta es el dato prioritario en la medición del paro, es el indicador admitido por la UE y el que se compara con el resto de Estados Miembros para medir el resultado global del mercado laboral a partir de la tasa de paro. ${ }^{3}$

No obstante, el PR desempeña un papel importante en el sistema estadístico español y europeo. Aunque no puede ni debe equipararse a la cifra de desempleo que ofrece la EPA, sí aporta elementos complementarios basados en la rapidez y bajo coste de su publicación, su periodicidad mensual, las posibilidades de estudio en ámbitos geográficos pequeños (municipal e inferior) o la posibilidad de seguimiento de los individuos a lo largo del tiempo. ${ }^{4}$ Pero no debemos olvidar que el fin

${ }^{2}$ En el informe de Africa y Lüdeke (2006) se compara detalladamente ambas cifras para Alemania, Austria, España, Finlandia y Suecia. Con anterioridad, White y Leyland (1992) ofrecen un repaso detallado de las diferencias entre países en el cálculo del desempleo. Para el caso concreto de España los trabajos de Malo (1999) y Pérez Infante (2000, 2006, 2008 y 2009) ofrecen un análisis minucioso de las discrepancias y de cuál ha sido su evolución en los últimos años (muy condicionada por los importantes cambios metodológicos que se han producido en ambas estadísticas).

${ }^{3}$ La EPA es la única estadística que estima a la vez todas las variables relacionadas con la situación de las personas en el mercado de trabajo (actividad, inactividad, ocupación y paro), además de cumplir estrictamente todos los requisitos establecidos a nivel internacional por la OIT en relación con el empleo y el paro.

${ }^{4}$ La Comisión Europea analiza también la situación del paro en los 27 países miembros mediante la tasa de paro armonizada. Esta tasa se calcula mensualmente con datos desestacionalizados con base en las encuestas trimestrales de población activa de cada país pero también incluye información sobre las variaciones mensuales del paro registrado. No obstante, Eurostat no publica las cifras de PR por no ser comparables entre países y la propia Comisión Europea no aceptó las cifras del PR como indicador utilizable para los Planes Nacionales de Acción para el Empleo, por lo que en 1998 se introduce en España el concepto de Demandantes de Empleo no Ocupados (DENOS) con el objetivo de aproximar el concepto de paro registrado a los criterios de la OIT. La cifra de DENOS se obtiene sumando a los parados registrados los colectivos que la definición de la OIT considera parados y no la Orden Ministerial de 1985: demandantes de empleo 
principal del registro en las oficinas públicas de empleo es la intermediación laboral entre los ofertantes de puestos de trabajo y los trabajadores, no la inscripción de los trabajadores sin empleo que desean encontrarlo. El simple hecho de que los datos del PR tengan carácter administrativo provoca que la magnitud de sus cifras se vea condicionada por los propios criterios administrativos y contables de la inscripción en las oficinas. ${ }^{5}$

Sin embargo, en el caso de Galicia, las dudas sobre la fiabilidad de la estimación del desempleo se han concentrado en la EPA, no solo por los agentes sociales, que la han llegado a calificar como irreal, sino por la propia Administración Pública. Las críticas se han dirigido a que debido a los criterios de elaboración de la muestra y el tamaño de la misma, la estimación del desempleo infravalora el volumen real de desempleados. Como principal prueba se presentaba la irreconciliable diferencia entre el paro estimado por la EPA y la cifra de PR, y su contradictorio signo respecto a lo que ocurre con el conjunto de España. En 2011 el número de parados estimados por la EPA en Galicia fue en media de 228.300, mientras que el número de parados registrados llego hasta las 241.323 personas. Es decir el paro estimado supera en un $5 \%$ al paro EPA mientras que para el conjunto de España la situación es la contraria, el paro EPA fue en 2011 un 17,5\% superior al PR. En la práctica estas diferencias, que han llegado a ser muy superiores (en 2008 el PR era en Galicia un $40 \%$ superior al paro estimado), han puesto en duda la validez de la EPA para estimar el desempleo en la economía gallega. De hecho, el propio Consello Económico e Social (CES-Galicia) en su memoria de 2006 llega a afirmar que Galicia ignora su nivel de desempleo. La fuerte brecha entre las cifras de PR, que difunde el Ministerio de Empleo y Seguridad Social, y las de desempleo estimado, que elabora el INE, son un claro indicador de que es necesaria una explicación para aclarar esta situación y

coyuntural, demandantes de empleo con jornada reducida inferior a 20 horas semanales y los trabajadores eventuales.

${ }^{5}$ La mayoría de expertos apuestan por la fiabilidad de los datos de la EPA e incluso hay quienes creen que el dato del PR por sí mismo no tiene validez, en gran medida porque su definición ha sufrido tantas alteraciones que es difícil saber a quien se considera hoy un parado registrado. Desde esta posición más crítica el PR se considera un concepto burocrático que se emplea con fines propagandísticos y que maquilla el volumen de desempleados. No obstante, no todo son criticas, ya que el PR permite conocer el desempleo incluso por municipios de residencia del trabajador, mientras que la muestra de la EPA tiene problemas de significatividad estadística sobre todo cuando se desagrega por provincias. 
demanda un mayor esfuerzo a la Administración para ajustarse a la dinámica sociolaboral gallega. ${ }^{6}$

La conciliación de ambas estadísticas sobre desempleo es un tema delicado al que se ha dedicado tiempo y esfuerzo tanto desde la Administración como desde la academia. Con anterioridad a los últimos cambios metodológicos en ambas estadísticas, el problema parecía resuelto tras el informe del Consejo Superior de Estadística sobre estadísticas coyunturales del mercado de trabajo (Consejo Superior de Estadística, 2002). El propósito del informe era intentar conciliar, hasta donde fuera posible, las cifras publicadas por la EPA con las provenientes de las fuentes administrativas. Para ello, se analizó el procedimiento utilizado en la elaboración de ambas estadísticas con el fin de intentar detectar los posibles factores que pudieran incidir en las diferencias observadas. En lo que se refiere al PR, el informe pone de manifiesto que la forma de obtener esta cifra está abocada a producir cifras inferiores a las de la EPA, ya que se obtiene deduciendo del número de demandantes de empleo a toda una serie de colectivos cuya situación de desempleo o cuya disponibilidad real para trabajar es discutible. Así pues, las diferencias entre las dos formas de medir el paro se atribuyen básicamente a que son conceptualmente diferentes. Para intentar conciliar las cifras, habría que recurrir a magnitudes conceptualmente homogéneas. Una forma de conseguirlo consiste en calcular un "paro registrado corregido" al que se añadan los colectivos excluidos que pueden cumplir con los requisitos de la EPA. Los resultados de ese ejercicio realizado con las cifras de 2001 son claros: apenas existen diferencias entre el número de parados que según la EPA están inscritos en el INEM 7 y el concepto de "paro registrado corregido". En todo caso, la conclusión de todo este análisis es unívoca: la cifra de paro que publica la EPA no puede descalificarse en base a las cifras del PR publicadas por el SPEE. ${ }^{8}$

${ }^{6}$ En el periodo 2005-2011, Galicia muestra el mayor desfase entre ambas medidas, lo que se plasma en que el paro estimado por la EPA solo suponga el 77\% de la cifra de PR. Galicia es el caso más extremo, pero no el único en el que sorprende la divergencia de datos. Así, en Navarra el paro EPA es el 81\% del PR; en Asturias, el 87\%; en Cantabria, el 86\%; y en País Vasco el 88\%. Notar que todas estas CC.AA. se distinguen por una intensidad de la inmigración inferior a la media nacional. Para el conjunto de España, el diferencial promedio es insignificante; el PR superó al paro EPA entre 2005 y 2008 pero desde entonces, debido al efecto de la mala coyuntura económica, el paro EPA supera en más de un $10 \%$ al PR.

7 Por la Ley 30/2003, se sustituye el INEM por los Servicios Públicos de Empleo, Estatal y de las Comunidades Autónomas.

${ }^{8} \mathrm{La}$ explicación realizada puede no ser tan adecuada tras los cambios metodológicos realizados en la EPA en 2002 y 2005 y en el PR en 2005. Como se explica detalladamente en Pérez Infante (2009) estos cambios reducen el nivel de desempleo 
Sin embargo, en Galicia, las dudas sobre la fiabilidad de los datos de desempleo de la EPA se repiten con bastante insistencia sobre todo desde los cambios metodológicos incorporados en 2002 y 2005 . A pesar de que trabajos aplicados a la economía gallega habían llegado a una conclusión similar a la obtenida en el informe del Consejo Superior de Estadística (véase Colino y Fernández, 2007) ${ }^{9}$, la presión social y mediática influyeron decisivamente en que desde la Administración autonómica se realizasen importantes esfuerzos para resolver esta situación. En primer lugar, el Instituto Galego de Estatística (IGE) coordinó una nueva operación estadística en colaboración con la Consellería de Traballo con el objeto de conocer la realidad sociolaboral de las personas inscritas en las oficinas públicas de empleo que se denominó "Enquisa aos Demandantes de Emprego" (Encuesta a los Demandantes de Empleo, EDE). Esta encuesta confirmó que el 45\% de los parados registrados en Galicia debían ser considerados como inactivos según los criterios de la OIT, que son los seguidos por la EPA. Es decir, se confirmaba que muchos parados registrados no se consideran como parados EPA, lo que revalida que las diferencias obtenidas entre ambas estimaciones del desempleo son perfectamente validas y consistentes. En segundo lugar, el propio IGE llegó a un acuerdo con el INE para que Galicia doblase su muestra en la EPA hasta los 8.600 hogares, lo que según los promotores de la ampliación permitiría hacer una estimación del desempleo más ajustada a la realidad. Los resultados obtenidos muestran que las importantes diferencias entre el paro EPA y el PR aunque persisten, tienden a reducirse. Esta aproximación entre ambas cifras, sin embargo, parece ser más fruto del ciclo económico que de la ampliación de la muestra de la EPA, ya que el paro estimado por la EPA aumenta en las fases recesivas a un ritmo superior al PR. Estos hechos sugieren que la explicación del diferencial observado entre ambas fuentes estadísticas en el caso gallego no reside en la "reducida" muestra de la EPA, tal y como se había afirmado por especialistas y agentes sociales.

estimado por la EPA en 2005 en aproximadamente 540.000 personas e incrementan el PR en 453.000. Consecuentemente el PR supera desde ese año al paro estimado por la EPA hasta que con la crisis económica la relación vuelve a invertirse.

${ }^{9}$ Colino y Fernández (2007) confirman que aunque los datos de la EPA y del SPEE son diferentes no son contradictorios y que el mayor diferencial observado en la economía gallega no es un problema de fiabilidad de la EPA en Galicia sino que puede deberse a una situación socioeconómica y demográfica particular, alejada de los valores medios nacionales. Los importantes cambios metodológicos en ambas fuentes impiden asegurar que los datos de paro estimado y PR muestren tendencias divergentes en distintos momentos del tiempo. Tampoco está justificado que la cobertura del PR deba ser homogénea por regiones. 
El principal objetivo de este artículo es examinar cómo se mide el desempleo en España y evaluar las diferencias entre las dos estimaciones de desempleo existentes para el caso del mercado de trabajo gallego. ${ }^{10}$ Para ello, en primer lugar se realiza una valoración del conjunto de fuentes estadísticas que existen en Galicia y España para medir el desempleo y se valora la evolución de sus diferencias en la última década. A continuación se analiza con detalle las diferencias existentes entre el paro estimado por la EPA y el PR utilizando la información suministrada por los microdatos de la propia EPA y de la EDE, una operación estadística única del IGE. Por último, al hilo de lo expuesto en los apartados anteriores se presentan algunas reflexiones sobre el tema en cuestión.

\section{El desempleo en Galicia: Fuentes estadísticas y evolución de las diferencias}

\subsection{Fuentes estadisticas}

En Galicia, como en el conjunto de España, hay dos estadísticas oficiales que miden periódicamente la evolución del paro: la EPA que elabora el INE y el PR en las oficinas públicas de empleo que publica mensualmente el SPEE. Entre estas dos estadísticas existen importantes diferencias metodológicas, pero sobre todo conceptuales, lo que se traduce en cifras dispares tanto para el conjunto de la economía española como a escala de las CC.AA. y que en algún caso, como en el gallego, han llegado a cuestionar la validez de la EPA para aproximar la evolución real del desempleo. Hay una diferencia esencial entre las dos medidas que se debe al propio objetivo que persiguen: el INE a través de la EPA trata de medir todas las situaciones en las que se encuentra una persona en relación con el mercado de trabajo (es decir, activo o inactivo, y ocupado o parado), mientras que el objetivo del SPEE es llevar un registro (de carácter

10 A pesar de los múltiples esfuerzos realizados, las diferencias entre ambas cifras siguen generando confusión especialmente en los debates políticos y económicos. De hecho, el último estudio de conciliación realizado por el Grupo de Trabajo de Estadísticas Coyunturales del Mercado Laboral (http://www.ine.es/daco/daco42/daco4211/compa_paro_2009.pdf) concluye que es necesario "continuar trasladando a los distintos ámbitos que la conciliación de cifras de paro asociadas a ambas fuentes de información no es posible, debido a que corresponden a conceptos distintos". 
administrativo) de los demandantes de empleo para instrumentar, entre otras, las políticas de ayuda al desempleo. ${ }^{11}$

\section{La Encuesta de Población Activa (EPA)}

La EPA se realiza trimestralmente por el INE sobre una muestra inicial de 65.000 hogares, conforme a los criterios de la OIT y de la Oficina Estadística de la UE (Eurostat). Los datos se recogen por entrevista personal y telefónica a personas en edad de trabajar y sus resultados se publican al cabo de un mes de finalizar el trabajo de campo. Se trata de una estimación y no de una estadística administrativa como en el caso del PR. En la EPA se consideran paradas a las personas entre 16 y 74 años que durante la semana de referencia han estado sin empleo, disponibles para trabajar y buscando activamente empleo. Según el Reglamento (CE) 1897/2000 existen nueve métodos de búsqueda de empleo considerados como "activos". Entre éstos se incluyen, por ejemplo, el estar en contacto con una oficina de empleo pública o privada con el fin de encontrar trabajo, enviar una candidatura directamente a los empleadores, o anunciarse o responder a anuncios de periódicos. Estas actividades deben haberse realizado en las cuatro semanas anteriores a la entrevista. En concreto, se tiene en cuenta a los que no tienen empleo por cuenta propia o ajena en la semana previa a la entrevista, buscan trabajo en las cuatro semanas anteriores, están disponibles para trabajar en las dos semanas siguientes o no buscan empleo porque ya han encontrado uno al que se incorporarán en los próximos tres meses. Por último, se considera parado a quien se ha inscrito en la oficina de empleo en las cuatro semanas previas a la entrevista, pero se excluye a los que sólo acuden a ella para renovar la demanda o acudir a cursos de formación ocupacional - estos dos últimos casos se clasifican como inactivos.

A lo largo de los años, las estimaciones ofrecidas por la EPA se han criticado por diversas causas -bien porque la gente no refleja adecuadamente su situación laboral en las encuestas (en muchas ocasiones porque las personas entrevistadas no disponen de información suficiente sobre la situación laboral de todos los miembros de su hogar), por la deficiente estimación de la estructura por edades, por la sub/sobreestimación de algunos colectivos como los inmigrantes, por el fortísimo crecimiento del empleo o por la continua ruptura de las series- lo que ha situado a los problemas de medición del desempleo crónicamente como titular en todos los medios de comunicación.

\footnotetext{
11 Para un análisis detallado de la medición del paro por parte de las dos estadísticas,
} véase Pérez Infante (2000) y Giráldez (2001). 
De lo que tampoco hay duda, es de los esfuerzos dedicados desde las distintas Administraciones Públicas a su mejora y perfeccionamiento, que durante los últimos años han sido constantes. De hecho, la EPA ha sido objeto de cuatro reformas desde 1999. En este año se ampliaron tanto el período de entrevistas como la muestra, con el fin de dar más continuidad a la encuesta. Paralelamente, se introdujeron modificaciones en el concepto de «subempleo». En 2000 se procedió a una actualización de las secciones muestrales, un cambio puramente técnico para adaptar la muestra a la estructura territorial de la población. En 2002 se produjo una nueva reforma, más profunda, y que persiguía tres objetivos. En primer lugar, una acomodación de los factores de elevación (que son los que se utilizan para, a partir de los datos de la muestra, conocer los que corresponden a la población), con el fin de dar una mayor ponderación a las edades centrales en la muestra, y que estaba motivada por la llegada de inmigrantes. En segundo lugar, depurar las distorsiones causadas por la falta de respuesta en las encuestas. Por último, adaptar la encuesta a la definición de desempleo propuesta por Eurostat, es decir, aplicar el Reglamento 1897/2000 y que tiene extrema importancia para explicar las diferencias observadas entre ambos métodos de cálculo del desempleo. Desde 2002, para considerar desempleado a una persona cuyo único método de búsqueda de empleo sea el registro en las oficinas de los servicios públicos de empleo debe haber tenido contacto con las mismas en las últimas cuatro semanas con el fin de encontrar trabajo (no vale la renovación de la demanda de empleo, la consulta de su prestación o el interés por acudir a cursos de formación). La EPA se vuelve a modificar en 2005. Como en 1999 y 2002 la mayoría de los cambios vienen impuestos por la nueva normativa europea (Reglamento 2527/2003), pero también se aprovechó para adecuarla a la nueva realidad demográfica y laboral (especialmente al aumento del número de extranjeros residentes). El reglamento, por su parte, exigía la incorporación de nuevas variables, y por lo tanto, de nuevas preguntas en el cuestionario lo que acabó traduciéndose en una casi completa renovación de éste (simplificando preguntas, cambiando su orden, modificando su redacción o variando las respuestas) así como en una modernización del sistema de recogida de la información. ${ }^{12}$

12 Aunque esta última reforma ha tenido una menor repercusión agregada sobre las cifras de desempleo que la de 2002, su impacto sobre los datos regionales ha sido muy heterogéneo. Mientras que tras el cambio en 2002 las cifras de desempleo se reducen en todas las CC.AA., en 2005 en algunas aumenta (por ejemplo, en Madrid en un 18\%) mientras que en otras se reduce considerablemente (como es el caso de Galicia en un $16 \%)$. 


\section{El Paro Registrado en el SPEE}

El Sistema Nacional de Empleo tiene como principal objetivo facilitar la inserción y promoción de los trabajadores en el mundo laboral para lo que se realizan diferentes acciones tendentes a mejorar su ocupabilidad. Para ello el trabajador debe formalizar una demanda de empleo en la oficina correspondiente a su domicilio que deberá renovar periódicamente. Esta demanda es necesaria para que los centros de empleo gestionen la inserción, la reinserción o la promoción laboral del trabajador.

El Sistema de Información de los Servicios Públicos de Empleo (SISPE) ${ }^{13}$, común a toda España, permite integrar la información relativa a la intermediación laboral que llevan a cabo los Servicios Públicos de Empleo, estatal y autonómicos. Dentro de la operación estadística Movimiento Laboral Registrado, mensualmente se ofrece información sobre demandantes de empleo, puestos de trabajo ofrecidos para gestión y colocaciones desagregadas por distintas variables y ámbitos geográficos (nacional, comunidades autónomas y provincias) que corresponde a la explotación del fichero de demandantes inscritos en las oficinas de empleo, en relación al último día laborable de cada mes.

La condición de demandante no equivale a la de trabajador en paro. Demandantes son todos los trabajadores que se inscriben en los servicios públicos de empleo para la búsqueda de un empleo o mejorar el que ya poseen (intermediación laboral), para recibir otros servicios ajenos al empleo (orientación para el autoempleo, formación ocupacional,....) o por la obligatoriedad de inscribirse para percibir una prestación contributiva o un subsidio. Por lo tanto, no todas las demandas de empleo registradas forman parte del concepto de PR. En concreto, el PR está constituido por el total de demandas de empleo existentes el último día de cada mes, excluyendo las que correspondan a situaciones laborales descritas en la Orden Ministerial de 11 de Marzo de 1985 (B.O.E. de

\footnotetext{
13 El SISPE sustituye en 2005 al SILE (Sistema de Información Laboral de Empleo), lo que sin modificar la Orden Ministerial de 11 de marzo de 1985 ha supuesto tres cambios importantes en el cálculo del PR: inclusión de los extranjeros demandantes de empleo no ocupados (anteriormente se excluían), actualización de la clasificación de los demandantes de empleo (afecta especialmente a estudiantes excluidos aunque ya finalizasen sus estudios, al no actualizarse su situación) y el cruce del registro de demandantes con el de afiliaciones a la Seguridad Social en situación de alta laboral (desde este momento, en los dos sentidos posibles; con lo cual si un demandante que estaba ocupado pierde su empleo se incluye en el PR en cuanto se produzca su baja como afiliado). Todos estos cambios supusieron un incremento del PR con datos de enero de 2005 de 453.050 personas (Pérez Infante, 2008 y 2009).
} 
14/3/85) por la que se establecen criterios estadísticos para la medición del PR. Para la clasificación de demandantes según el colectivo de pertenencia y calcular por exclusión la cifra de PR, al poder reunir la demanda de un trabajador inscrito dos o más situaciones, como se indica en la introducción metodología de la Estadística de Empleo, se ha establecido el siguiente orden de preferencia contabilizándose un demandante en el primer colectivo que aparece (ver Cuadro 1).

\section{Cuadro 1. Clasificación de Demandas de Empleo en el SPEE}

\begin{tabular}{|c|c|c|c|}
\hline $\begin{array}{l}\text { DEMANDANTES SIN } \\
\text { DISPONIBILIDAD } \\
\text { Demandan servicios ajenos } \\
\text { al empleo o no tienen } \\
\text { disponibilidad }\end{array}$ & & $\begin{array}{r}\text { Demanda } \\
\text { Demar } \\
\text { Demandante }\end{array}$ & $\begin{array}{l}\text { tes de otros servicios ajenos al empleo } \\
\text { Demandantes de autoempleo } \\
\text { lantes sin disponibilidad inmediata } \\
\text { en suspensión sin intermediación laboral }\end{array}$ \\
\hline \multirow{22}{*}{$\begin{array}{l}\text { DEMANDANTES DE } \\
\text { EMPLEO } \\
\text { Demandan empleo por } \\
\text { cuenta ajena y tienen } \\
\text { disponibilidad para el } \\
\text { empleo }\end{array}$} & \multirow{21}{*}{$\begin{array}{l}\text { Demandantes de } \\
\text { empleo no } \\
\text { parados }\end{array}$} & \multirow{3}{*}{$\begin{array}{l}\text { Baja } \\
\text { disponibilidad o } \\
\text { atención } \\
\text { específica }\end{array}$} & Demandantes en suspensión con intermediación laboral \\
\hline & & & Demandantes jubilados o mayores de 65 años \\
\hline & & & Demandantes pensionistas \\
\hline & & \multirow{6}{*}{$\begin{array}{l}\text { Ocupados o con } \\
\text { relación laboral }\end{array}$} & $\begin{array}{l}\text { Perceptores de prestaciones adscritos en trabajos de colaboración } \\
\text { social }\end{array}$ \\
\hline & & & $\begin{array}{l}\text { Demandantes que están en suspensión o reducción de jornada por } \\
\text { un expediente de regulación de empleo (ERE) }\end{array}$ \\
\hline & & & Demandantes con contrato de fijo discontinuo. \\
\hline & & & $\begin{array}{l}\text { Demandantes Trabajadores eventuales agrarios subsidiados } \\
\text { (TEAS) }\end{array}$ \\
\hline & & & $\begin{array}{l}\text { Afiliados a la Seguridad Social regímenes generales ó de } \\
\text { autónomos }\end{array}$ \\
\hline & & & Ocupados regímenes agrícolas \\
\hline & & \multirow{7}{*}{$\begin{array}{l}\text { Demandantes de } \\
\text { un empleo } \\
\text { específico }\end{array}$} & Demandantes de trabajo sólo a domicilio \\
\hline & & & Demandantes de teletrabajo \\
\hline & & & Demandantes de trabajo sólo para el extranjero \\
\hline & & & Demandantes emigrantes con voluntad de regreso \\
\hline & & & $\begin{array}{l}\text { Demandantes de trabajo sólo en otra comunidad distinta a la de } \\
\text { residencia }\end{array}$ \\
\hline & & & Demandantes que exportan la prestación al extranjero \\
\hline & & & Extracomunitarios con condiciones específicas de acceso al empleo \\
\hline & & \multirow{5}{*}{$\begin{array}{l}\text { Demandantes no } \\
\text { ocupados }\end{array}$} & $\begin{array}{l}\text { De trabajo por un período inferior a tres meses (empleo } \\
\text { coyuntural) }\end{array}$ \\
\hline & & & De jornada inferior a 20 horas semanales (a tiempo parcial) \\
\hline & & & $\begin{array}{l}\text { Estudiantes menores de } 25 \text { años o que demandan primer empleo } \\
\text { si tienen más de } 25 \text { años }\end{array}$ \\
\hline & & & De servicios previos al empleo \\
\hline & & & Demandantes con alta especial en Seguridad Social \\
\hline & $\begin{array}{l}\text { Demandantes de } \\
\text { empleo parados }\end{array}$ & $\begin{array}{l}\text { PARO } \\
\text { REGISTRADO }\end{array}$ & RESTO DE DEMANDANTES \\
\hline
\end{tabular}

Fuente: Elaboración propia. 


\section{Cuadro 2: Clasificación de los Demandantes Inscritos (SPEE). Septiembre 2012. Total España y Galicia}

\begin{tabular}{|c|c|c|c|c|c|}
\hline & \multirow{3}{*}{$\begin{array}{c}\text { TOTAL } \\
\text { DEMANDANTES }\end{array}$} & \multicolumn{3}{|c|}{$\begin{array}{c}\text { DEMANDAS PENDIENTES } \\
\text { NO INCLUIDAS PARO REGISTRADO }\end{array}$} & \multirow{2}{*}{ PARO REGISTRADO } \\
\hline & & & & DENOS & \\
\hline & & OCUPADOS & $\begin{array}{l}\text { CON } \\
\text { DISPONIBILIDAD } \\
\text { LIMITADA } \\
\end{array}$ & $\begin{array}{l}\text { OTROS NO } \\
\text { OCUPADOS/TEAS }\end{array}$ & PARO REGISTRADO \\
\hline ESPAÑA & 6.276 .407 & 976.866 & 236.230 & 358.032 & 4.705 .279 \\
\hline GALICIA & 342.904 & 44.547 & 22.498 & 16.486 & 259.373 \\
\hline
\end{tabular}

Fuente: Elaboración propia.

Gráfico 1. Relación entre Paro EPA y Paro Registrado

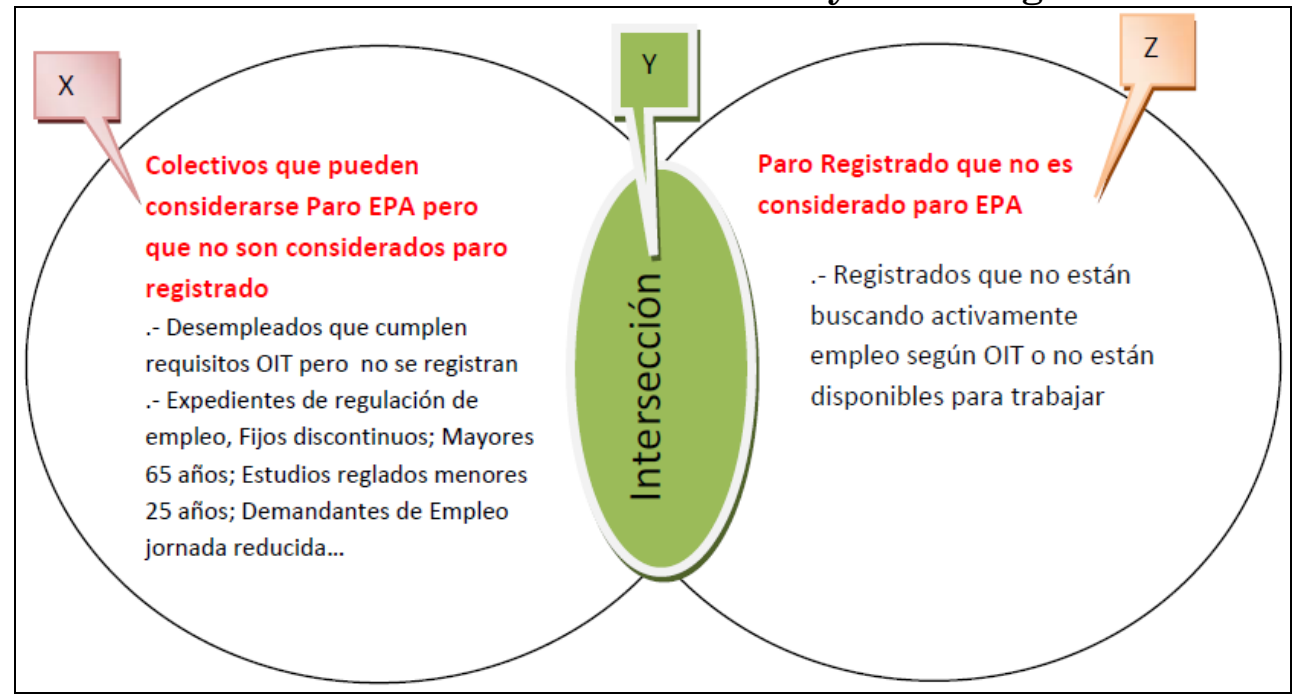

Fuente: Elaboración propia.

Con la Ley sobre Infracciones y Sanciones en el Orden Social de 1988 se añadió como criterio de exclusión del cómputo del PR a los demandantes que rechazasen acciones de inserción laboral adecuadas a sus características, según se establece en el Art. 17 apartado 2 del Real Decreto Legislativo 5/2000, de 4 de Agosto (Rechazo de acciones de inserción laboral). En los últimos años se produjo una aplicación más estricta de las exclusiones, lo que en muchos casos ha sesgado al alza el número de personas excluidas. El último cambio relevante se produjo en 2008 cuando se excluyeron del registro del desempleo a algunas de las 
personas que acudían a cursos de formación, que se catalogaron como DENOS.

En septiembre de 2012, 6.276.407 personas estaban inscritas como demandantes activos de los cuales 342.904 residían en Galicia (ver Cuadro 2 ). De este total, aproximadamente el $75 \%$ son reconocidos como parados registrados. Los demandantes excluidos del PR figuran bajo la denominación "Demandas pendientes no incluidas en el paro registrado" que incluye todas las situaciones anteriormente descritas que pueden agruparse en tres grandes bloques: Demandantes ocupados, Demandantes con disponibilidad limitada y Otros no ocupados y Trabajadores eventuales agrarios subsidiados (TEAS), grupos que podrían ser considerados como parados por la EPA.

Concluyendo, estamos ante dos cifras que se complementan, pero representan a dos colectivos distintos (ver Gráfico 1). El número total de desempleados EPA seria $\mathrm{U}_{\mathrm{EPA}}=\mathrm{X}+\mathrm{Y}$. El número total de desempleados registrados es $\mathrm{UPR}_{\mathrm{PR}}=\mathrm{Y}+\mathrm{Z}$.

Por lo tanto, estas dos cifras (UEPA y UPR) pueden diferir considerablemente, ya que son dos grupos de personas que solo coincidirían de forma parcial. Incluso en el caso de que ambas cifras fuesen parecidas, las dos categorías de desempleo serian realmente diferentes ya que solo el colectivo representado por Y (desempleados EPA que figuran registrados en las oficinas del servicio público de empleo y cumplen las características de la Orden Ministerial para considerarse un $\mathrm{PR})$ pertenece a ambas categorías. El hecho de que una cifra sea superior a otra depende de un amplio conjunto de elementos que pueden variar con el tiempo. Por lo tanto, es tan normal que el paro EPA sea superior al PR como lo contrario (Pérez Infante, 2009). Lo primero ha ocurrido desde 1977 salvo en tres años (2005-2007) periodo que se inicia con la implantación del SISPE y la reforma de la EPA (ver nota a pie de página 7).

\subsection{Análisis de las diferencias en la cuantificación del desempleo gallego.}

El primer inconveniente a la hora de realizar este ejercicio es la falta de homogeneidad temporal, tanto por los diferentes periodos de referencia (mensual/trimestral) como por el elevado número de cambios metodológicos realizados en ambas estadísticas. Respecto al primer problema, dado que la EPA proporciona estimaciones de parados por trimestres y que el PR en el SPEE tiene carácter mensual, se calcula un PR trimestral como la media aritmética (simple) de cada trimestre. El segundo problema solo tiene solución en la medida que tanto el INE como 
el SPEE traten de reconstruir sus series para poder realizar comparaciones temporales homogéneas.

\section{Gráfico 2: Evolución del Número de Parados en Galicia y España (1996T1-2011T4)}

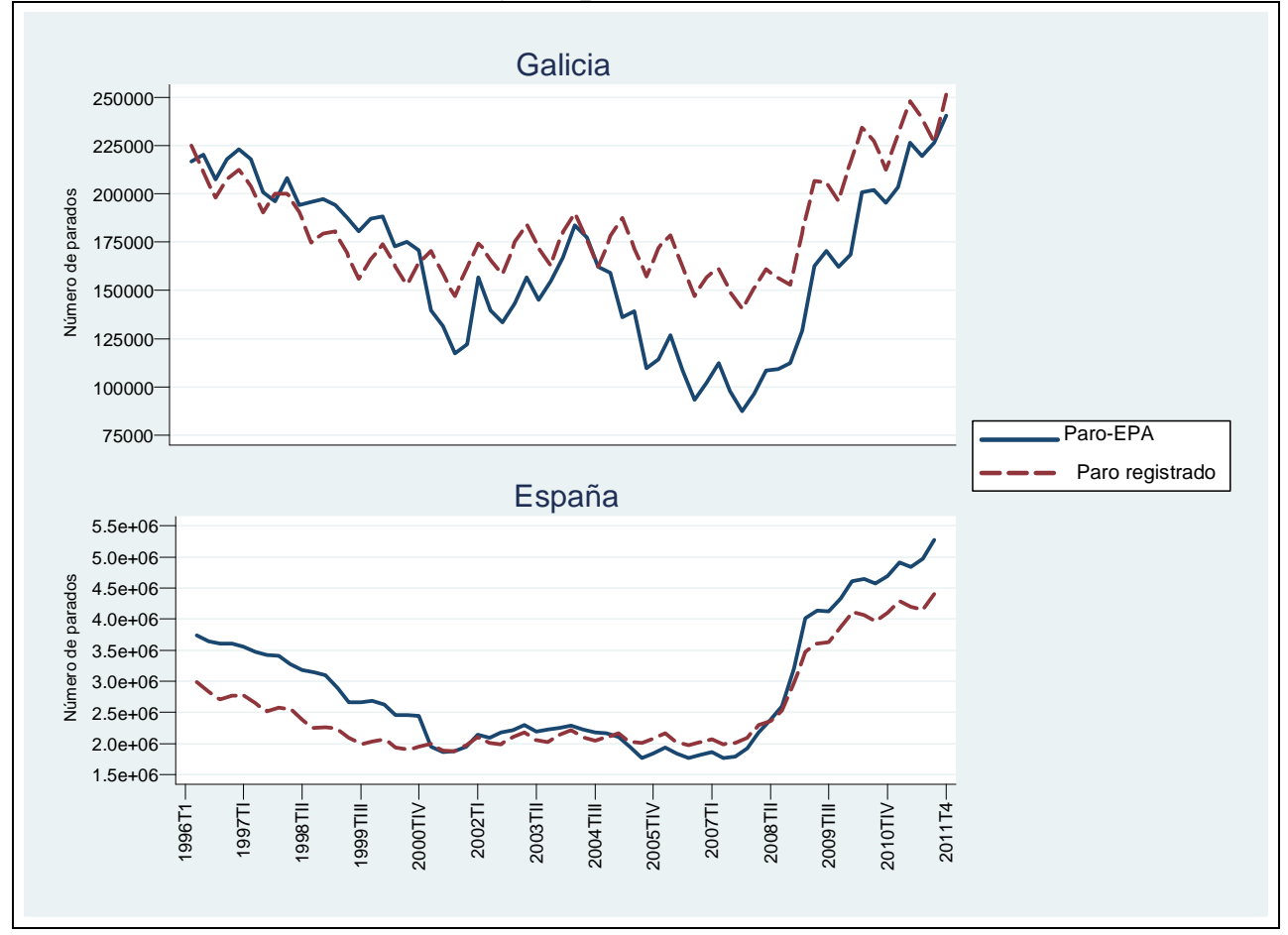

Fuente: Elaboración propia con datos de la EPA y del SPEE.

En el Gráfico 2 mostramos la evolución entre los años 1996 y 2011 del paro estimado por la EPA (dato trimestral) y del PR $^{14}$ (promedio

14 El primer año completo en que comienza la serie homogénea de PR es 1996. Los datos de PR previos a 2005 (anteriores por tanto a la implantación del SISPE) están reconstruidos con el sistema SISPE. Hasta 2001 esa reconstrucción se hizo con datos individuales, con lo que su homogeneidad con los posteriores a 2005 es total. Sin embargo, las reconstrucciones de 1996 a 2000 están basadas en correcciones de datos agregados y, aunque comparables, nunca puede ser lo mismo que el cómputo del paro registrado con el sistema SISPE con datos individuales. Para un análisis del proceso de obtención de las series y su impacto sobre la reconstrucción de los datos del periodo 2001-2005 consultar el trabajo de Toharia y Malo (2005) y para el periodo 1996-2000 el informe de Toharia y Pérez Infante, "Enlace de los datos de paro registrado a partir del 
trimestral de los datos mensuales), para Galicia y España. Prácticamente en todos los años se producen notables discrepancias entre ambas cifras de paro, resultado que como hemos visto no debe sorprender ya que se trata de dos indicadores que aproximan conceptos laborales similares pero no coincidentes. En Galicia, las diferencias son muy significativas; en especial desde 2005 el PR publicado por el SPEE permanece sistemáticamente por encima del estimado por la EPA. Durante el periodo 1996-2001, la relación era totalmente opuesta y es desde el 2001 que el PR supera al paro EPA, situación que se acentúa desde el último cambio metodológico en ambas estadísticas en 2005.

La relación entre ambas cifras es muy diferente en el caso de España. En el periodo 1996-2001, al igual que en el caso gallego, el paro EPA es superior al PR siguiendo una senda similar. A partir de 2001, ambas cifras son muy próximas (aunque se mantiene el mayor valor para el paro EPA), hasta que con el doble cambio metodológico de 2005, el PR supera ligeramente al paro EPA. Desde el inicio de la actual crisis económica la relación se invierte y el paro estimado por la EPA supera claramente al PR.

Las diferencias entre ambas series y su diferente comportamiento en España y Galicia pueden observarse de manera más nítida en el Gráfico 3 donde se muestra la evolución temporal de la tasa de cobertura del PR —definida como PR en porcentaje de paro estimado- entre 1996 y 2011. Podemos apreciar claramente tres periodos.

En el primero, entre el primer trimestre de 1996 y el primer trimestre de 2001, la tasa de cobertura se mantiene aproximadamente constante y en torno al 80\% para España y algo mayor en el caso de la economía gallega. Tras el cambio en 2002, comienzan los problemas para la economía gallega al incrementarse de forma considerable la cobertura del PR, mientras que para el conjunto de la economía española las diferencias entre ambas estadísticas se reducen considerablemente. Tras el cambio metodológico en 2005, la diferencia entre ambas fuentes es muy exagerada en la economía gallega y comienza a reducirse tras los últimos cambios en el registro del paro en 2008, y los previsibles efectos de la crisis económica.

sistema SILE y el sistema SISPE” (versión de septiembre de 2005): http://www.sepe.es/contenido/estadisticas/datos estadisticos/empleo/datos/estim antigu as/ConvenioUAH SISPESILE.pdf. 


\section{Gráfico 3: Evolución de la Tasa de Cobertura del PR en España y Galicia (1996T1-2011T4)}

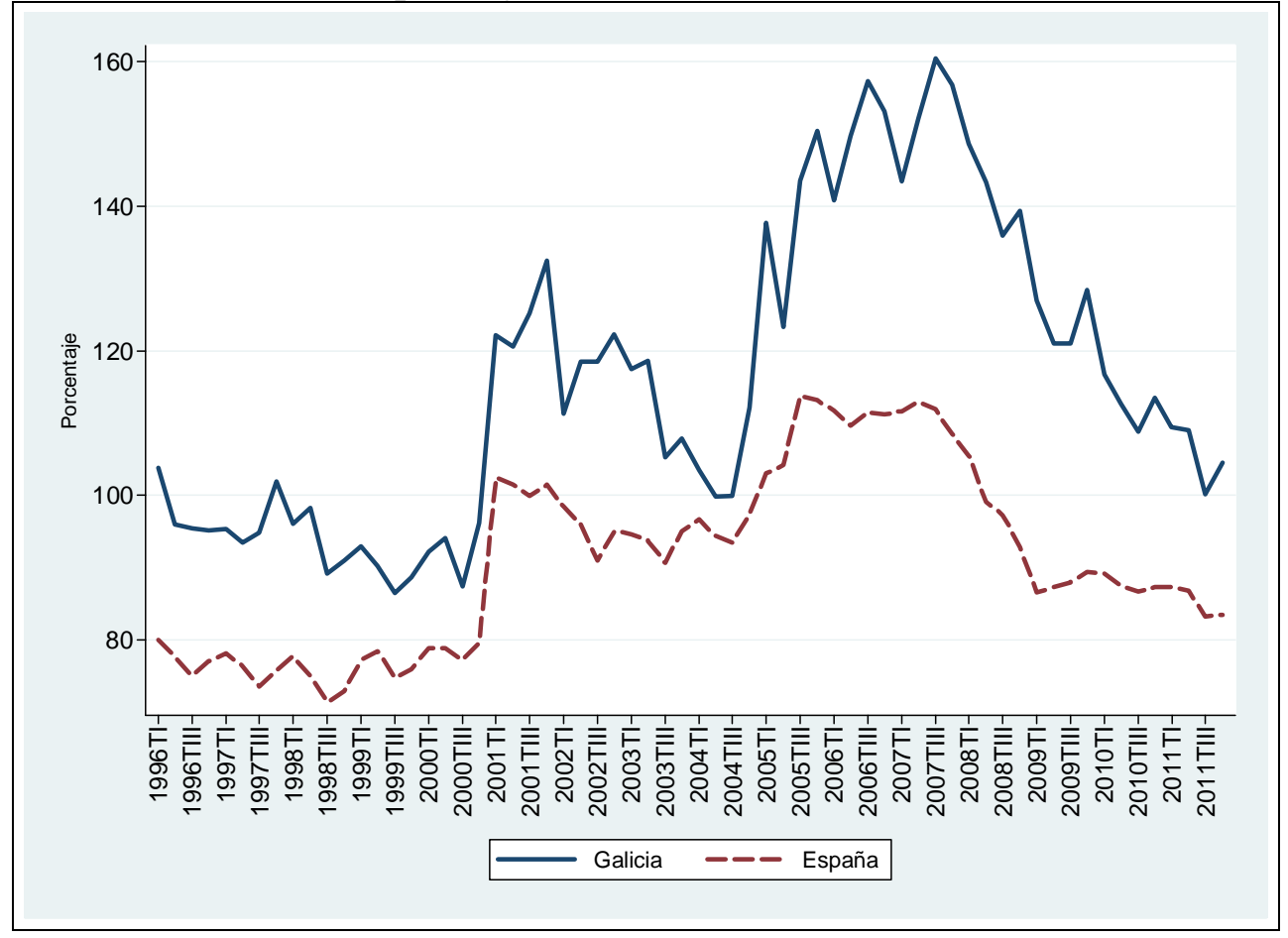

Fuente: Elaboración propia con datos de la EPA y del SPEE.

¿A qué se debe esta diferencia en la cifra final de desempleados? La respuesta está motivada por las distintas metodologías y colectivos analizados. Según Pérez Infante (2006, 2008), hay dos razones fundamentales que explican que sea mayor la cifra de la EPA a la del PR, aunque también puede darse la situación inversa. Primero, no todos los parados de la EPA buscan trabajo en las oficinas de los servicios públicos de empleo estatal y autonómico, sino que recurren a agencias privadas, anuncios, contactos personales o envío de currículos. En segundo lugar, no todas las personas que se inscriben como demandantes en un registro público están paradas según los criterios del PR. Para la EPA estos últimos sí contarían si reúnen los requisitos necesarios -estar desocupados, buscar empleo y estar disponibles para trabajar- pero no para el PR. Este sería el caso, por ejemplo, de estudiantes menores de 25 años o de quienes buscan empleos de corta duración o de jornada reducida. Entonces, lo esperable es que el paro EPA sea superior al PR, que es lo que ocurre para España y Galicia entre 1996 y 2001. A partir de este año el 
paro EPA es inferior al PR en casi todos los trimestres en el caso de Galicia y en menos de la mitad en el caso de España, lo que refleja los cambios en la definición de parado según la EPA (desde 2005, la renovación de la demanda de empleo en el registro no demuestra que se esté buscando empleo, lo que reduce el número de desempleados EPA y mantiene el número de parados registrados). Con la crisis actual, sin cambio de criterio alguno, el paro EPA vuelve a ser claramente superior al registrado en España (no así en Galicia). ${ }^{15}$ A pesar de que las diferencias se han ido reduciendo paulatinamente, muchos expertos siguen cuestionando la falta de fiabilidad estadística del PR porque no incluye al total de demandantes de empleo no ocupados (DENOS), con lo que estaría subestimando la cifra real de parados (lo que en el caso de Galicia situaría las diferencias entre ambos conceptos en valores superiores a los actuales, pero aproximaría las cifras en el conjunto de la economía española).

\section{3. ¿Por qué es la diferencia entre ambos conceptos de paro tan elevada en Galicia?}

Antes de adentrarnos en el análisis de las características de las personas inscritas en los servicios públicos de empleo según la Enquisa aos demandantes de emprego (EDE), conviene examinar, la capacidad de la EPA para aproximar el número de inscritos en las oficinas públicas de empleo. En el Cuadro 3 se compara la cifra total de inscritos publicada por el SPEE, incluidos los "demandantes de otros servicios", y la cifra del total de inscritos en las oficinas públicas de empleo que podemos estimar a partir de los microdatos de la EPA. Los cálculos realizados muestran una sobrestimación del número de inscritos en los servicios públicos de empleo por la EPA, superior en el caso de España que en el caso de Galicia. Desde 2007 las diferencias son en media inferiores al 10\% y no ofrecen discrepancias significativas entre ambas economías que permitan explicar el elevado diferencial observado en la tasas de cobertura (ver Gráfico 3).

Dado que en muchas ocasiones quien responde al cuestionario de la encuesta en el hogar (que es la unidad muestral de la EPA) no conoce al

15 Un elemento esencial para explicar este último cambio es la baja inscripción de inmigrantes en el registro del paro, que puede notarse sobre todo en las épocas de crisis. La inclusión de los extranjeros en el PR es muy reducida. Además, en todas las CC.AA. el paro estimado de la población no nacional en la EPA supera claramente al PR de ese colectivo, lo que puede explicar en parte las diferencias entre la cobertura del PR por CC.AA. (dada las importantes diferencias entre territorios en la incidencia de la inmigración). Sin embargo, también desde 2009 el paro estimado entre los nacionales supera al PR, salvo en algunas CC.AA. entre las que destaca Galicia con el mayor diferencial absoluto. 
detalle la situación de todos sus miembros, este es un resultado que podríamos considerar normal ya que existe una tendencia a clasificar como inscrito a todos los parados o individuos que estén buscando empleo. Para ambas economías la correlación entre las tasas de variación anual de ambas series es muy elevada (0,97 en Galicia y 0,98 en España), lo que confirma la fiabilidad de la EPA en este punto. En cualquier caso, con las salvedades apuntadas, parece deducirse que la EPA constituye un instrumento adecuado para estudiar el comportamiento de las personas inscritas en los servicios públicos de empleo.

Centrémonos ahora en la composición y en el comportamiento de los inscritos en los servicios públicos de empleo según la EPA y la EDE a la hora de buscar empleo. Para ello, analizamos primero el comportamiento de los inscritos en las oficinas públicas de empleo según la información suministrada por los microdatos de la EPA.

\section{Cuadro 3: Número Total de Inscritos en los Servicios Públicos de Empleo en España y Galicia según Estimaciones de la EPA y según el SPEE (2005TIII-2011TIV)}

\begin{tabular}{|c|c|c|c|c|c|c|}
\hline & \multicolumn{3}{|c|}{ Galicia } & \multicolumn{3}{|c|}{ España } \\
\hline & EPA & SPEE & (EPA-SPEE)/SPEE & EPA & SPEE & (EPA-SPEE)/SPEE \\
\hline 2005TIII & 244.036 & 210.009 & $16,20 \%$ & 3.604 .293 & 3.005 .904 & $19,91 \%$ \\
\hline 2005TIV & 248.720 & 218.372 & $13,90 \%$ & 3.602 .114 & 3.040 .314 & $18,48 \%$ \\
\hline $2006 \mathrm{TI}$ & 252.335 & 220.716 & $14,33 \%$ & 3.752 .066 & 3.094 .886 & $21,23 \%$ \\
\hline 2006TII & 251.652 & 209.239 & $20,27 \%$ & 3.615 .018 & 2.950 .325 & $22,53 \%$ \\
\hline 2006TIII & 229.697 & 199.519 & $15,13 \%$ & 3.631 .610 & 2.963 .886 & $22,53 \%$ \\
\hline 2006TIV & 227.843 & 202.874 & $12,31 \%$ & 3.493 .667 & 2.972 .694 & $17,53 \%$ \\
\hline $2007 \mathrm{TI}$ & 222.917 & 202.794 & $9,92 \%$ & 3.523 .310 & 3.021 .975 & $16,59 \%$ \\
\hline 2007TII & 216.424 & 195.470 & $10,72 \%$ & 3.266 .926 & 2.961 .932 & $10,30 \%$ \\
\hline 2007TIII & 213.333 & 188.851 & $12,96 \%$ & 3.247 .343 & 3.023 .490 & $7,40 \%$ \\
\hline 2007TIV & 208.388 & 195.611 & $6,53 \%$ & 3.345 .868 & 3.064 .897 & $9,17 \%$ \\
\hline $2008 \mathrm{TI}$ & 223.523 & 202.381 & $10,45 \%$ & 3.488 .828 & 3.232 .396 & $7,93 \%$ \\
\hline 2008TII & 217.435 & 205.153 & $5,99 \%$ & 3.611 .548 & 3.389 .294 & $6,56 \%$ \\
\hline 2008TIII & 228.971 & 204.825 & $11,79 \%$ & 3.884 .639 & 3.642 .971 & $6,63 \%$ \\
\hline 2008TIV & 248.623 & 230.211 & $8,00 \%$ & 4.406 .384 & 4.087 .209 & $7,81 \%$ \\
\hline 2009TI & 266.821 & 258.089 & $3,38 \%$ & 5.102 .678 & 4.662 .478 & $9,44 \%$ \\
\hline 2009TII & 287.693 & 268.258 & $7,24 \%$ & 5.433 .644 & 4.923 .887 & $10,35 \%$ \\
\hline 2009TIII & 284.245 & 261.385 & $8,75 \%$ & 5.567 .224 & 5.052 .359 & $10,19 \%$ \\
\hline 2009TIV & 296.576 & 279.712 & $6,03 \%$ & 5.816 .888 & 5.293 .750 & $9,88 \%$ \\
\hline 2010TI & 314.692 & 294.518 & $6,85 \%$ & 6.070 .937 & 5.519 .449 & $9,99 \%$ \\
\hline 2010TII & 320.512 & 294.513 & $8,83 \%$ & 6.027 .072 & 5.503 .051 & $9,52 \%$ \\
\hline 2010TIII & 304.593 & 279.610 & $8,94 \%$ & 5.950 .798 & 5.441 .215 & $9,37 \%$ \\
\hline 2010TIV & 326.099 & 294.788 & $10,62 \%$ & 6.115 .907 & 5.535 .663 & $10,48 \%$ \\
\hline 2011TI & 335.090 & 308.779 & $8,52 \%$ & 6.240 .136 & 5.694 .738 & $9,58 \%$ \\
\hline 2011TII & 333.807 & 309.191 & $7,96 \%$ & 6.210 .097 & 5.665 .286 & $9,62 \%$ \\
\hline 2011TIII & 332.272 & 300.666 & $10,51 \%$ & 6.294 .157 & 5.668 .717 & $11,03 \%$ \\
\hline 2011TIV & 353.161 & 322.411 & $9,54 \%$ & 6.610 .089 & 5.913 .521 & $11,78 \%$ \\
\hline
\end{tabular}

Fuente: Elaboración propia con datos de la EPA y del SPEE. 


\section{Cuadro 4: Clasificación de los Inscritos en las Oficinas de los Servicios Públicos de Empleo como Ocupados, Parados o Inactivos (en porcentajes) en España y Galicia según la EPA} (2005-2011)

\begin{tabular}{|c|c|c|c|c|c|c|}
\hline & \multicolumn{4}{|c|}{ Galicia } & \multicolumn{3}{c|}{ España } \\
\cline { 2 - 7 } & $2005-2007$ & $2008-2011$ & $2005-2011$ & $2005-2007$ & $2008-2011$ & $2005-2009$ \\
\hline Ocupados & $22 \%$ & $15 \%$ & $17 \%$ & $24 \%$ & $13 \%$ & $17 \%$ \\
Parados & $38 \%$ & $52 \%$ & $47 \%$ & $36 \%$ & $61 \%$ & $52 \%$ \\
Inactivos & $40 \%$ & $33 \%$ & $36 \%$ & $40 \%$ & $26 \%$ & $31 \%$ \\
\hline
\end{tabular}

Fuente: Elaboración propia con datos de la EPA.

Tal y como indicamos en el Cuadro 4, no existen diferencias significativas en relación a la situación laboral de los demandantes de empleo inscritos en ambas economías según estima la EPA cuando consideramos el periodo 2005-2007 completo. En ambos casos, solo en torno al $37 \%$ de los inscritos son considerados parados según la EPA, mientras que un $23 \%$ se consideran ocupados. El restante $40 \%$ son considerados inactivos. No obstante, en el último periodo considerado (2008-2011) sí existen diferencias significativas entre el comportamiento estimado en España y Galicia. En ambos casos, el porcentaje de inscritos considerados ocupados ha descendido significativamente (siete puntos porcentuales en Galicia y casi doce en España) lo que en parte puede explicar la aproximación entre el PR y el paro EPA desde ese año para ambas economías. En este último periodo el porcentaje de inscritos considerados parados EPA en España paso del 36\% al $61 \%$ mientras que en Galicia no supera el 52\%, más de 9 puntos porcentuales inferior. Por lo tanto, parece confirmarse que el diferencial entre ambos conceptos debe ser claramente superior en el caso gallego, pero ¿cuál es la razón última?

Las diferencias, como hemos indicado, se circunscriben principalmente a la situación de parado-inactivo. ${ }^{16}$ Recordemos que desde 2002 no es válida la simple inscripción en las oficinas públicas de empleo como método de búsqueda activa y por eso pueden darse mayores diferencias. Parece, por tanto, que la búsqueda activa de empleocaracterística necesaria para ser considerado parado EPA-no se cumple en un número importante de los individuos inscritos en las oficinas públicas de empleo según estima la EPA. ¿Pero hasta qué punto es válida

16 Las diferencias entre los ocupados inscritos estimados por la EPA en Galicia y los Registrados ocupados desde 2007 son inferiores a un 4\%, que podemos atribuir al error de muestreo. 
esta interpretación para explicar las diferencias de cobertura del PR? ¿Y qué dificulta la búsqueda activa de empleo en mayor medida en Galicia que en el conjunto de España? ${ }^{17}$

\section{Cuadro 5: Efectos Marginales sobre la Probabilidad de Ser Parado-EPA para las Personas Inscritas en las Oficinas Publicas de Empleo en Galicia según la EPA (1) y la EDE (2)}

\begin{tabular}{|l|c|c|}
\hline Variables & $(1)$ & $(2)$ \\
\hline Provincia (Ourense=0) & 0,077 & 0,028 \\
A Coruña & 0,124 & 0,026 \\
Lugo & $0,238^{* * *}$ & $0,066^{* *}$ \\
Pontevedra & & \\
Sexo (hombre=0) & $-0,213^{* * *}$ & $-0,154^{* * *}$ \\
Mujer & & \\
Edad (16-29=0) & 0,062 & $-0,038$ \\
$30-44$ & $-0,201^{* * *}$ & $-0,166^{* * *}$ \\
$45-59$ & $-0,380^{* * * *}$ & $-0,395^{* * * *}$ \\
60 y más & & \\
Estado civil (Viuda/o=0) & $-0,043$ & 0,049 \\
Soltera/o & $-0,059$ & $-0,01$ \\
Casada/o & 0,103 & 0,146 \\
Divorciada/o & 773 & 4225 \\
\hline $\mathrm{N}^{\circ}$ observaciones & 0,108 & 0,072 \\
Pseudo-R & $87,600(0,000)$ & $361,590(0,000)$ \\
Wald chi2(10) $(p$-value) & & \\
\hline
\end{tabular}

Niveles de significación: $* * * p<0.01 * * p<0.05 * p<0.10$.

Para contestar a ambas preguntas, a continuación realizamos un estudio del perfil de los individuos inscritos y considerados parados e inactivos según la EPA en Galicia y lo comparamos con el perfil obtenido a partir de la EDE. Dado que la EDE tiene como referencia temporal el 31 de Octubre de 2008 seleccionamos el cuarto trimestre de la EPA de ese año para realizar la comparación. Queremos examinar, por tanto, en qué medida ser parado EPA se asocia con una serie de características del

17 Aunque la distinta intensidad de la búsqueda de empleo parece la explicación más plausible a estas diferencias, también podría ser relevante una distinta disponibilidad para trabajar (que es otro de los requisitos exigidos por la OIT para que una persona pueda ser considerada como parada). 
individuo como son el sexo, la edad, el estado civil, y la provincia de residencia. Para ello estimamos dos modelos logit por Máximo Verosimilitud (véase Cameron y Trivedi, 2005) utilizando los microdatos de la EPA y la EDE. En las columnas 1 y 2, respectivamente, del Cuadro 5 mostramos los efectos marginales (promedio) de las variables explicativas sobre la probabilidad de ser parado tipo EPA. ${ }^{18}$

De la primera estimación (columna 1), que utiliza los microdatos de la EPA, obtenemos dos importantes conclusiones: ser mujer y superar los 45 años se asocia muy positivamente con ser inactivo en la EPA. En concreto la probabilidad aumenta muy considerablemente cuando se supera los 60 años, lo que puede atribuirse a que en este tramo de edad muchos de los inscritos en las oficinas públicas de empleo lo hacen como situación intermedia a la jubilación. Residir en la provincia de Pontevedra, en cambio, se asocia positivamente con ser parado tipo EPA (en relación con Ourense); esto nos podría estar indicando que la dispersión de la población y el tipo de actividad principal incide negativamente en la búsqueda activa de empleo. ${ }^{19}$ Por lo tanto, allí donde existan más mujeres entre los registrados, mayor sea el porcentaje de inscritos con edades superiores a los 45 años y mayor sea la dispersión geográfica de la población en edad de trabajar, la diferencia entre el PR y el paro EPA será mayor. Estas conclusiones se confirman con los resultados de la

\footnotetext{
${ }_{18}$ En ambas estimaciones la variable dependiente es de tipo binaria y toma el valor 1 o 0 según la persona se considere, respectivamente, parada o inactiva según los criterios de la EPA. Como variables explicativas, hemos incluido aquellas características personales del individuo que están disponibles tanto en la EPA como en la EDE, que son el sexo, la edad (con variables binarias para cuatro grupos de edad: 16-29 años, 3044 años, 45-59 años, y 60 y más años), y el estado civil (con cuatro variables binarias según la persona sea viuda, soltera, casada o divorciada). También se incluyeron variables ficticias para las provincias gallegas que pretenden captar factores geográficos como la dispersión de la población o el tipo de actividad principal (por ejemplo, en Lugo, según la Encuesta Movilia 2006 del Ministerio de Fomento, el 14\% de los trabajadores tienen como lugar de trabajo su propio domicilio frente al $2 \%$ para el conjunto de España). Entre las variables explicativas no hemos considerado la nacionalidad porque, aunque sí se incluye en el cuestionario de la EDE, no está disponible en los microdatos.

${ }_{19}$ Galicia concentra alrededor de la mitad de las entidades singulares y más del 50\% de los diseminados de España. Aproximadamente el 15,6\% de la población gallega vive en diseminado, lo que supone una realidad única (para el conjunto del Estado solo el 3,4\% de la población vive en diseminados). Con más de 27.600 núcleos con menos de 100 habitantes donde viven aproximadamente el 22,5\% de la población, es fácil intuir las dificultades para brindar cualquier servicio público de proximidad como los ofrecidos por las oficinas públicas de empleo. En Pontevedra el peso en la población total de las personas que viven en núcleos con menos de 100 habitantes es aproximadamente el 17\% ligeramente inferior al peso en Coruña (20\%), Ourense (25\%) y por supuesto Lugo (41\%).
} 
estimación realizada con los datos de la EDE (columna 2). Al igual que antes, la probabilidad de ser parado EPA disminuye con la edad (a partir de los 45 años), es menor para las mujeres, pero mayor para las personas residentes en la provincia de Pontevedra. ${ }^{20}$

\section{Cuadro 6: Relación con la Actividad según los Criterios de la} OIT (\% sobre el Total de Parados Registrados)

\begin{tabular}{|c|c|c|c|}
\hline & Total & Hombres & Mujeres \\
\hline Parados registrados clasificados como ocupados & 8,8 & 9,0 & 8,6 \\
\hline Parados registrados clasificados como parados & 45,8 & 53,9 & 40,2 \\
\hline Parados registrados clasificados como inactivos & 45,4 & 37,1 & 51,2 \\
\hline $\begin{array}{c}\text { Con responsabilidades familiares (cuidado de niños, } \\
\text { adultos enfermos, discapacitados, ... }\end{array}$ & 13,3 & 4,0 & 19,9 \\
\hline Con enfermedad o incapacidad & 7,4 & 7,2 & 7,5 \\
\hline Cursando estudios & 5,1 & 5,0 & 5,2 \\
\hline En otra situación (incluye a los desanimados) & 19,6 & 20,9 & 18,7 \\
\hline
\end{tabular}

Fuente: Elaboración propia con datos de la EDE (2008).

En el Cuadro 6 profundizamos en el perfil de los parados registrados a partir de los datos de la EDE. Según esta encuesta solo el $45,8 \%$ de los parados registrados cumplen los criterios para definirse como parado según la OIT. Un porcentaje similar el $45,4 \%$ son considerados como inactivos por diferentes razones entre las que destaca estar desanimados (19,6\%). Este resultado supone directamente compatibilizar las estimaciones realizadas a partir de la EPA con las obtenidos en el PR. Si del total de parados registrados nos quedamos con el $45,8 \%$, la cifra de PR corregido en el cuarto trimestre de 2008 sería de 82.309 personas a las que deberíamos sumar los otros (6.784) para obtener una cifra de "paro registrado" comparable con el paro estimado por la EPA. Esta operación situaría el total de parados estimados según los criterios de la OIT en 89.094 personas claramente inferior a la cifra estimada por la EPA (112.444). Esta diferencia aunque importante (20\%) puede considerarse aceptable, ya que al aplicar los criterios de la OIT para la definición de parado a las personas inscritas solo estaríamos obteniendo una estimación

20 Si nos fijamos en la parte inferior del Cuadro 5 vemos que el valor del pseudo- $\mathrm{R}^{2}$ no es muy elevado aunque el test de Wald de significatividad global del modelo rechaza la hipótesis de nulidad de todos los parámetros para cualquier nivel de significación estadística. 
parcial del paro real (el estimado en la EPA) ya que son muchas las personas (sobre todo inmigrantes ${ }^{21}$ y jóvenes) que no contemplan como medida de búsqueda de empleo acudir a las oficinas públicas de empleo.

Por último, tal y como se muestra en el Cuadro 7, en la mayor parte de los casos la inactividad se justifica por la realización de tareas del hogar y la atención de otras responsabilidades familiares. En este caso cabe destacar las enormes diferencias por sexo, que se traducen finalmente en la no consideración de muchas mujeres como paradas según los criterios de la OIT.

Cuadro 7: Parados Registrados Considerados Inactivos según su Situacion de Inactividad (\%)

\begin{tabular}{|l|c|c|c|}
\hline & Total & Hombres & Mujeres \\
\hline Realizando tareas del hogar & 40,7 & 16,7 & 52,8 \\
\hline Atendiendo a otras responsabilidades familiares & 14,9 & 9,4 & 17,7 \\
\hline Preparando oposiciones & 5,0 & 4,4 & 5,2 \\
\hline Estudiando & 4,8 & 6,5 & 3,9 \\
\hline Prejubilado & 2,1 & 4,7 & 0,8 \\
\hline Otra situación & 32,6 & 58,3 & 19,5 \\
\hline
\end{tabular}

Fuente: Elaboración propia con datos de la EDE (2008).

\section{Conclusiones}

La existencia de varias fuentes estadísticas que ofrecen cifras desiguales sobre indicadores del mercado laboral tan importantes como el número de desempleados ha provocado siempre confusión en la ciudadanía, e incluso, usos inadecuados de las estadísticas oficiales por parte de los agentes sociales, periodistas y también políticos con responsabilidades de gobierno. El objetivo de este artículo era comprobar la fiabilidad de la EPA en la estimación del desempleo para el caso gallego, puesta en duda desde diversos ámbitos (Administración autonómica, partidos políticos, sindicatos, academia y medios de comunicación) por el elevado diferencial existente en Galicia entre el PR y los parados estimados por la EPA. La comparación de esta situación con la existente a nivel del conjunto de España, donde el diferencial además de ser de signo contrario era muy inferior cuantitativamente, generó un elevado consenso

${ }^{21}$ Los extranjeros son aproximadamente el $25 \%$ del paro estimado por la EPA, mientras que solo son el $10 \%$ del PR. 
entre los distintos agentes sociales sobre la idea de que "posiblemente" se estaba "maquillando" el desempleo gallego en las estimaciones ofrecidas por la EPA.

Desde nuestro punto de vista, no existía ninguna duda sobre la fiabilidad de la EPA como demostraron trabajos anteriores, pero la insistencia social ha supuesto que para aclarar esta confusión mediática fuese necesario ampliar la muestra de la EPA (con un desembolso directo solo para la realización de los trabajos relativos a la recogida de información de la muestra ampliada de 234 secciones superior a 500.000 euros, al que deben sumarse los costes directos e indirectos de personal y medios dedicados a esta operación por el propio IGE); y realizar una operación estadística nueva para conocer la situación laboral de los demandantes de empleo. La primera operación, por el momento, no ha servido para reducir el diferencial observado entre ambas cifras de desempleo, aunque sin duda, ha mejorado la estimación de todas las variables representativas de la situación laboral en Galicia. Sus resultados han servido para confirmar la validez de las anteriores estimaciones y evitar así las dudas sobre la fiabilidad de las cifras de desempleo estimadas a partir de la EPA. La segunda operación, con un coste y compromiso muy inferior, ofrece información muy relevante sobre la situación laboral de las personas inscritas en las oficinas públicas de empleo y ha permitido confirmar que el diferencial existente entre ambas estimaciones para el caso gallego se puede explicar básicamente porque una mayoría de los inscritos no buscan activamente empleo. ${ }^{22}$ En concreto, tres características de los inscritos inciden en que en Galicia el diferencial entre paro estimado y PR sea mayor que en el conjunto de España: la mayor participación de la mujer en el PR, el envejecimiento de la población activa $\mathrm{y}$, posiblemente, un hábitat poblacional muy disperso (recordemos que más del 50\% de los núcleos de población de España son gallegos, lo que sin duda dificulta la accesibilidad a los servicios públicos de empleo).

Por último, incidir una vez más en que ambas cifras y metodologías, EPA y SPEE, son importantes y complementarias para llegar a conclusiones consistentes y coherentes sobre aspectos relacionados

\footnotetext{
${ }^{22}$ Este es un elemento crucial para entender las diferencias entre ambas cifras. En el PR el único método de búsqueda contemplado es la propia inscripción en el registro, considerando el resto de métodos inadecuados para definir a un demandante de empleo como parado. Mientras, en la EPA, la búsqueda puede realizarse por métodos diferentes, entre los que se encuentra estar en contacto con la oficina del servicio público de empleo (que no debe entenderse como estar inscrito o solo ponerse en contacto para la renovación de la demanda).
} 
con el mercado de trabajo y la evolución del mismo y que, en vez de un problema, es una verdadera suerte contar con información tan detallada como la aportada por el SPEE. Desde aquí apostamos por una mayor utilización de la información suministrada por el SPEE para una mejor comprensión y estimación de la cifra de paro de Galicia y las distintas CC.AA, que operaciones como la "Enquisa aos demandantes de Emprego" pueden poner en valor.

\section{Bibliografía}

Africa, M. y Lüdeke, B., (2006): "Registered unemployment (RU) compared with harmonised unemployed (LFS)", European Commission Working Papers and Studies.

Cameron, A.C. y Trivedi, P.K., (2005): Microeconometrics, Methods and Applications, Cambridge University Press, New York.

Colino, J. y Fernández, M. (2007): Cantos parados hai en Galicia? Unha reflexión sobre a medición estatística do desemprego, Informe de Coxuntura da Economía Galega, tercer trimestre 2007, Fundación CaixaGalicia.

Comisión Europea (1999): "Registered unemployment and harmonised unemployment figures", Annex pp. 143-148, in Employment in Europe 1998.

Consejo Superior de Estadística (2002): "Comparación de datos de empleo y paro 2001", Grupo de trabajo sobre estadísticas coyunturales del mercado de trabajo.

Fernández, M. y Flores, M. (2012): "Diferencias entre Paro Registrado y Paro EPA en España: Una evaluación a partir de Microdatos de Galicia”. IRENe Working Papers $n^{0} 1$.

Giraldez, M.T. (2001): "La medición del paro en España y en la Unión Europea", en L. Fina y L. Toharia (Coordinadores), El empleo en España: situación y perspectivas, Ministerio de Trabajo y Asuntos Sociales, Madrid. 
Instituto Galego de Estatística (2009): Enquisa aos Demandantes de Emprego (EDE).

Instituto Nacional de Estadística: Encuesta de Población activa (EPA), varios años.

Instituto nacional de Estadística (2011): Comparación de las magnitudes estadisticas de Paro según la Encuesta de Población Activa y los datos de los Servicios Públicos de Empleo a partir de la vinculación de información de microdatos de ambas fuentes. Segundo trimestre 2009. (http://www.ine.es/daco/daco42/daco4211/compa_paro_2009.pdf).

Malo, M.A. (1999): Paro registrado: Análisis comparativo y enseñanzas para España, Informe elaborado para la Subdirección General de Promoción de Empleo del Instituto Nacional de Empleo.

Pérez Infante, J.I. (2000): "La medición del desempleo en España: la EPA y el paro registrado", Revista del Ministerio de Trabajo y Asuntos Sociales. Economía y Sociología, 21, pp. 19-57.

Pérez Infante, J.I. (2006): Las estadísticas del mercado de trabajo en España, Ministerio de Trabajo y Asuntos Sociales. Colección Informes y Estudios Empleo, Madrid.

Pérez Infante, J.I. (2008): “Por qué el paro registrado supera al paro de la EPA?", Revista del Ministerio de Trabajo e Inmigración. Economía y Sociología, 71, pp. 53-65.

Pérez Infante, J.I. (2009): "La medición del paro: el paro de la EPA y el paro registrado", Cuadernos de mercado de trabajo, Observatorio de las Ocupaciones del Servicio Público de Empleo Estatal, número 3, junio 2009.

Servicio Público de Empleo Estatal (SPEE): Series de demandantes y parados registrados, varios años.

Toharia, L. (2006): "A situación do mercado de traballo en Galicia", Informe de Coxuntura da Economía Galega, primer trimestre 2006, Fundación CaixaGalicia.

Toharia, L. y Malo, M.A. (2005): Influencia de la implantación del SISPE en el paro registrado, Servicio Público de Empleo Estatal (mimeo), junio. 
(http://www.sepe.es/contenidos/inicial/sispe/pdf/Docum1-Efecto_SISPE_01jun-05.pdf )

White, A. y Leyland, J. (1992): "How unemployment is measured in different countries", Employment Gazette, 100, pp. 421-431. 